\title{
Temporo-insular enhancement of EEG low and high frequencies in patients with chronic tinnitus. QEEG study of chronic tinnitus patients
}

\author{
Morteza Moazami-Goudarzi ${ }^{1,2^{*}}$, Lars Michels ${ }^{2}$, Nathan Weisz ${ }^{3}$, Daniel Jeanmonod ${ }^{2,4}$
}

\begin{abstract}
Background: The physiopathological mechanism underlying the tinnitus phenomenon is still the subject of an ongoing debate. Since oscillatory EEG activity is increasingly recognized as a fundamental hallmark of cortical integrative functions, this study investigates deviations from the norm of different resting EEG parameters in patients suffering from chronic tinnitus.

Results: Spectral parameters of resting EEG of male tinnitus patients ( $n=8$, mean age 54 years) were compared to those of age-matched healthy males ( $n=15$, mean age 58.8 years). On average, the patient group exhibited higher spectral power over the frequency range of 2-100 Hz. Using LORETA source analysis, the generators of delta, theta, alpha and beta power increases were localized dominantly to left auditory (Brodmann Areas (BA) 41,42, 22), temporo-parietal, insular posterior, cingulate anterior and parahippocampal cortical areas.

Conclusions: Tinnitus patients show a deviation from the norm of different resting EEG parameters, characterized by an overproduction of resting state delta, theta and beta brain activities, providing further support for the microphysiological and magnetoencephalographic evidence pointing to a thalamocortical dysrhythmic process at the source of tinnitus. These results also provide further confirmation that reciprocal involvements of both auditory and associative/paralimbic areas are essential in the generation of tinnitus.
\end{abstract}

\section{Background}

Tinnitus is an auditory phantom perception, reported subjectively as a tone and/or a noise, in the absence of an external stimulus [1]. Approximately $5-15 \%$ of the general population experience tinnitus [2]. In 1-3\% of the general population the tinnitus affects the quality of life, involving sleep disturbance, work impairment and psychological distress $[3,4]$. The underlying physiological mechanisms that lead to phantom sensation are still being explored. In most cases, tinnitus is accompanied by an audiometrically measurable hearing loss, and even in a majority of those cases with normal audiograms abnormal outer or inner hair-cell function has been reported correlating with the presence of tinnitus $[5,6]$.

Contemporary views of tinnitus emphasize the role of the central auditory system [1,4,7-14]. Studies in anaesthetized animals suggest enhanced firing rate and/or

\footnotetext{
* Correspondence: mormo@ini.phys.ethz.ch

${ }^{1}$ Institute of Neuroinformatics, ETHZ/UNIZH, Winterthurerstrasse 190, 8057 Zurich, Switzerland
}

synchronized firing to be a necessary neurophysiological mechanism underlying tinnitus $[9,15]$. A reduction of tinnitus intensity in patients has been correlated to reduction of delta band power [16].

Alterations in spontaneous central neuronal activity patterns after peripheral deafferentations have recently been proposed to be essential in the genesis of tinnitus $[6,17,18]$. A relevance for peripheral deafferentation has also been proposed in the field of neurogenic pain, which prompted some authors to envisage that a similar mechanism might be at the source of tinnitus and neurogenic pain [19-23]. Peripheral deafferentation leads to thalamic desactivation [24], which in turn disrupts normal thalamocortical (TC) interaction $[7,10]$, thus leading to the appearance of tinnitus. The effects of an abnormal thalamocortical interaction can be analysed at the cortical level using magnetoencephalogram (MEG) or electroencephalogram (EEG) $[7,25]$. This sequential view integrates both the induction in the periphery and the generation at the TC level of tinnitus. In the following,

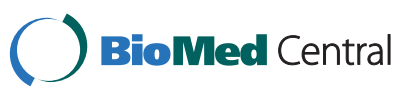


we refer to a mechanism that focuses on TC interplay [7]. First evidence for this mechanism in tinnitus was the finding of low-threshold calcium spike (LTS) bursts in the medial thalamus [10]. 50\% of neuronal activity in the medial thalamus (central lateral nucleus, CL) was characterized as LTS bursts. LTS bursts displayed a delta/theta rhythmicity, with a mean interburst discharge rate of $4 \mathrm{~Hz}$. LTSs have been described intracellularly in in vitro and in vivo experiments and have been related to a state of membrane hyperpolarization [26-28]. In tinnitus this would be a consequence of auditory deprivation caused by peripheral damage.

CL is part of the medial thalamus, is diffusely connected to wide cortical areas $[29,30]$ and is thought to serve as a non-specific amplifier of TC activity [31]. The TC loop constitutes an important component contributing to the rhythmicity of scalp EEG and MEG [7,32]. The $4 \mathrm{~Hz}$ discharge rate of thalamic LTS bursts may be supposed to attract the TC system into low EEG frequencies, a proposition which is at the base of this and other studies. Thus analyzing spectral features of continuously recorded EEG offers a window for the investigation of abnormal TC interplay in tinnitus patients. Surprisingly, even though spontaneous activity has been a frequent research target in animal models of tinnitus, studies in humans have been rare. A few studies have looked at the resting oscillatory EEG/MEG in tinnitus patients as compared to healthy controls [7,25,33-37]. In a resting EEG study of patients with severe tinnitus as compared to healthy controls a significant increase of Z- score power over the frequency ranges from 0.5 to $22 \mathrm{~Hz}$ was reported [37], which was dominant in fronto-temporal electrodes. Another EEG study described an increase and decrease of average total power in female and male patients as compared to healthy controls, respectively [38]. A further study of spontaneous brain activity reported temporal and fronto-temporal changes (increases and decreases) of relative power in individuals with severe tinnitus [36]. Most recently, an MEG study found an increase and decrease of delta and alpha power respectively in tinnitus patients as compared to healthy controls [25]. Further studies emphasized the relevance of gamma ( $>40 \mathrm{~Hz})$ activity to the pathophysiology of tinnitus $[34,35]$. The aforementioned findings have been incongruous, showing both increase and decrease in different frequency band power. Therefore the characteristic of different frequency bands remains an ongoing debate in the pathogenesis of tinnitus. Otherwise, attempts to study tinnitus in humans have focused on the use of designs that measure neurophysiological responses to sounds $[17,39,40]$ or experimental manipulations that enhance or reduce the perceived loudness [20,41-43]. In a recent experiment of that kind, Kahlbrock and Weisz [16] found transient reductions of tinnitus intensity following the offset of a masker (so-called residual inhibition, RI), accompanied by significant reduction in the delta frequency band. These changes were specific to a masker inducing RI and not observed with maskers that do not.

In light of the high variability of results we still lack sufficient knowledge of the anomalies of the resting EEG state in tinnitus patients, and the localization of cortical generators at the source of the observed power excesses has not been investigated in detail.

In the present study, we use power spectrum analysis and source localization of EEG data to identify cortical regions with changes in the underlying spontaneous activity patterns in individuals with tinnitus under both conditions eyes closed (EC) and eyes open (EO) as compared to healthy controls.

Our findings can be added to the microphysiological [10] and magnetoencephalographic $[7,24,25]$ evidence for a thalamocortical dysrhythmic process at the source of tinnitus, characterised by a low frequency overproduction in thalamocortical loops.

\section{Methods \\ Patients}

The patient group consisted of 8 male patients (mean age 54 yrs, range 41-70 yrs). Patient demographics are summarized in Additional file 1: Table S1. Patients were referred by otorhinolaryngologists after all typical treatment options had been applied (see disease duration in Additional file 1: Table S1). The patient group was therefore characterized by chronic therapy resistant and outspoken tinnitus mechanisms.

\section{Healthy controls}

The healthy control group consisted of 15 male subjects (mean age 58.8, 41-64 yrs). Statistical comparison revealed no statistical difference between the age of the healthy controls and the patient group ( $\mathrm{t}$-test, $\mathrm{p}=0.36$ ).

All control subjects were screened for health problems using a detailed health questionnaire (Zürcher Gesundheits-Fragebogen; Kuny and Stassen, 2004). They had no current or previous history of relevant physical illness and no current medication which could affect their EEG.

\section{EEG recording sessions}

The study was approved by the Kanton Zurich ethics committee. All subjects, patients and controls were informed about the aim and the scope of the study and all gave written informed consent according to the declaration of Helsinki. Subjects were seated in a dimly lit room shielded against sound and stray electric fields and were video-monitored. All EEGs were acquired in the morning between 9 and 12 in order to exclude an impact of circadian factors on the EEG. Recording 
sessions of patients and controls followed an interleaved schedule and the recording apparatus was continuously calibrated. Subjects refrained from caffeinated beverages on the day of recording to avoid a caffeine-induced theta decrease in EEG [44]. Since drowsiness may result in enhanced theta power, the vigilance of subjects was checked by monitoring EEG parameters, such as slowing of the alpha rhythm or appearance of spindles. In addition, at the end of the recording, subjects were asked if they were awake during the whole recording session. Within each session, spontaneous EEG was recorded under two conditions: while subjects rested with their eyes closed, and while they rested with their eyes open. EEG was recorded for 5 min under each condition. Before each recording, subjects were instructed to assume a comfortable position in a chair. They placed their head on a chin-rest in order to minimize headmovements. For EC condition, subjects were instructed to place their fingers on their eyelids to reduce rolling eye movements and to relax but to stay awake. For eyes open condition, subjects were requested to keep eyes open and to maintain gaze on a fixation mark on the wall of the recording chamber. EEG signals were measured using $60 \mathrm{Ag} / \mathrm{AgCl}$ surface electrodes, which were fixed in a cap at the standard positions according to the extended 10-20 system (Easycap, Herrsching, Germany). During recording, electrode $\mathrm{CPz}$ served as reference. Impedances were below $5 \mathrm{k} \Omega$ in all electrodes processed in the further analysis. We used two additional bipolar electrode channels as eye and electromyogram (EMG) monitors. EEG signals were registered using the SynAmps EEG system (Neuroscan Compumedics, Houston, TX, $0.017 \mathrm{uV}$ precision, sampling rate $250 \mathrm{~Hz}, 0.3-$ $100 \mathrm{~Hz}$ analog band pass filter, $-12 \mathrm{~dB}$ /octave) and continuously viewed on a PC monitor.

\section{Data preprocessing and editing}

Data were analysed offline using Matlab (The Matworks, Natick, MA), EEGLAB (http://sccn.ucsd.edu/eeglab[45]), and custom scripts. The scalp EEG was re-referenced to the mean of the signals recorded at the ear lobes. Data were inspected in $5 \mathrm{~s}$ epochs, and artifacts from large muscle or eye movement were removed. For editing purposes, muscle artifacts were considered significant if the underlying EEG rhythms were not clearly seen. The EEG was decomposed into independent components using blind separation (independent component analysis, ICA). After the removal of components containing eye movement, muscle artifacts or heart beats, the signal was reconstructed. This procedure resulted in at least 143s of EEG per subject for estimates of power spectral density (mean: $278 \pm 73$ s).

To investigate the effect of ICA component rejection, we compared tinnitus and control power spectra for EC condition in two approaches: 1) after visual artefact rejection only (before ICA) and 2) after additional ICA component rejection (after ICA). To test for significant differences between the two approaches we performed a repeated-measure ANOVA, considering mean band powers as within-subject variables and groups as between-subject variables. The mean power in the delta $(2-4 \mathrm{~Hz} ; \mathrm{F}=4.00, \mathrm{p}=0.06)$, theta $(4-8 \mathrm{~Hz} ; \mathrm{F}=2.00$, $\mathrm{p}=0.17)$, alpha $(8-12 \mathrm{~Hz} ; \mathrm{F}=0.75, \mathrm{p}=0.39)$, low beta $(12-18 \mathrm{~Hz} ; \mathrm{F}=1.55, \mathrm{p}=0.22)$, high beta $(18-30 \mathrm{~Hz}$; $\mathrm{F}=1.86, \mathrm{p}=0.18)$, and gamma $(30-100 \mathrm{~Hz} ; \mathrm{F}=1.88$, $\mathrm{p}=0.18$ ) frequency bands did not show a statistically significant difference between the two approaches. Therefore, we continue by reporting the results of ICA corrected data.

\section{Power spectral analysis}

The spectral analysis was performed with the multitaper method, which offers optimal spectral smoothing, and allows the trading of resolution in the frequency domain for reduced variance and has been described in details elsewhere (http://www.chronux.org[46]). Fourier transform was applied to tapered time series signal. We used an optimal family of orthogonal tapers (slepian functions). These are parameterized by their time length $\mathrm{T}$ and frequency bandwidth $\mathrm{W}$. For chosen $\mathrm{T}$ and $\mathrm{W}$, maximally $\mathrm{k}=2 \mathrm{TW}-1$ tapers well centred in frequency are appropriate for spectral estimation. Power spectra were estimated by time-bandwidth product $\mathrm{TW}=4$ and $k=3$ tapers. The choice of the parameters depends on the available data set and could be made by iterative visual inspection [46]. In order to normalize the power spectra for each subject, the relative quantitative changes of power $(\Delta \mathrm{P})$ for each electrode were calculated by subtracting the average of the power spectra for each electrode of the subject for the EC condition.

\section{Statistical analysis of spectra}

For groupwise comparisons, the power spectra of the tinnitus group and the healthy control group were compared with a non-parametric Wilcoxon rank sum test for each frequency point; Z-values were not corrected for multiple comparisons and were therefore considered as exploratory for frequency point comparisons.

For band power comparison, we applied a false discovery rate (FDR) [47] correction for multiple comparisons. The corrected significant $\mathrm{p}$-values were verified to have at least $\mathrm{p}<0.05$.

\section{LORETA imaging}

To localize the cortical sources of scalp EEG activity we used low resolution electromagnetic tomography analysis (LORETA; http://www.unizh.ch/keyinst[48]). The LORETA method is a discrete, three-dimensional (3D) 
distributed, linear, inverse solution. The smoothing constraints in LORETA endows the tomography with the property of low localization errors to test point sources, albeit with low spatial resolution (i.e. neighbouring neuronal sources will be highly correlated). The description of the method can be found in [48]. The LORETA inverse solution corresponds to the $3 \mathrm{D}$ distribution of electrical neuronal activity that features a maximum similarity (i.e. maximum synchronization), in term of orientation and strength, between neuronal populations in adjacent voxels. The imaging is therefore particularly tuned towards synchronized brain activities as they occur, e.g. in spreading oscillatory activations. Since LORETA takes explicitly into account that scalp electric potentials are determined up to an arbitrary additive constant, the final LORETA solution is independent of the electrical reference used. It is worth emphasizing that deep structures such as the anterior cingulate cortex [49], and mesial temporal lobes [50] can be correctly localized with LORETA. In the implementation of LORETA, computations were made in a head model using the MNI305 template [51], with the three-dimensional solution space restricted to cortical gray matter, as determined by the probabilistic Talairach atlas [52]. Voxel size is $7 \mathrm{~mm}$ on each side. Quantitative neuroanatomy (including Brodmann areas) were determined by the probabilistic Talairach atlas [52]. Anatomical labels such as Brodmann areas are also reported using MNI space, with correction to Talairach space [53]. In order to calculate tomographic LORETA images we first calculated the cross-spectral density matrix using multitaper FFT. With a spatial over-smoothing of $10^{-4}$ the current source density was estimated for 2394 cortical voxels within the frequency bands given above [54]. This procedure resulted in one 3D LORETA image for each subject for each frequency range.

LORETA images were statistically compared between groups through multiple voxel-by- voxel comparisons in a nonparametric test for functional brain imaging [55]. The $\mathrm{t}$-values corresponding to $\mathrm{p}<0.01$ or better were plotted onto a MRI template with a scale bar indicating statistical power and colour scale linearity equal to 75 .

\section{Results}

\section{Mean power spectra}

In order to summarize the data and because spectra from all electrodes demonstrated similar shape and scale, we averaged the log-transformed spectra of all scalp electrodes for each subject. We then averaged these individual spectra to one spectrum for the patient group and one spectrum for the control group (Fig. 1A, $B)$. The patient group exhibited more spectral power over the frequency range $(2-100 \mathrm{~Hz})$ for both the EO and EC conditions. The distribution of band power across the patient and control groups was significantly higher $(\mathrm{p}<0.02)$ in the delta, theta and beta frequency bands, with slightly stronger effects for EO condition. Significant differences in delta, theta, and beta frequency bands $(\mathrm{p}<0.02)$ were also demonstrated between patients and controls for the EC condition, both before and after normalization (see Additional file 2: Figure $\mathrm{S} 1)$. Therefore, the increase in band power is unlikely to be due to a DC increase in power, which would be diminished or suppressed after normalization.

To investigate if there is any difference between patients with and without hyperacusis (see Additional file 1: Table S1), we performed a statistical unpaired two-sample $t$-test. There were no significant differences in the delta $(\mathrm{p}=0.91)$, theta $(\mathrm{p}=0.72)$, alpha $(\mathrm{p}=0.60)$, beta1 $(\mathrm{p}=0.88)$, beta2 $(\mathrm{p}=0.92)$, and gamma $(\mathrm{p}=0.72)$ spectral band power between the two subgroups.

\section{Scalp power topography}

After establishing a significant difference between patient and control group in spectra averaged over all electrodes, we were interested to know which electrodes contributed most to this difference and at which frequency. We performed Wilcoxon rank sum tests for each electrode at each frequency point and plotted the matrix of Z-values (Fig. 2A, Fig. 3A). Consequently we applied FDR correction for multiple comparisons for mean band power between the two groups, and significant electrodes were marked with black circles. At a higher statistical significance, delta increases were shown in left centro-temporo-parietal regions for the EC condition (corrected $\mathrm{p}<0.004$ ), and lateralized to the left more than right in the EO condition (corrected $\mathrm{p}<0.0019)$. Theta increases were dominantly localized to the left temporo-parietal and right centro-parietal areas (corrected $\mathrm{p}<0.016$ ), in the EC condition, and left temporo-parieto-central regions (corrected p < 0.001 ) in the EO condition. The main beta increase is localized to left fronto-centro-parietal regions for the EC condition (corrected $\mathrm{p}<0.05$ ), and right fronto-central regions for the EO condition (corrected $\mathrm{p}<0.05)$.

\section{LORETA source localization}

Generators of differential power are shown as activation in statistical maps for different frequency ranges (Fig. 4, 5). The enhanced spectral EEG power of the patient group is reflected in cortical overactivation in delta, theta, alpha and beta frequency ranges focused over following cortical areas: auditory (BA 41, 42, 22), temporoparietal (BA 21, 40), insular posterior, cingulate anterior (BA 24, 32), parahippocampal, and prefrontal/premotor (BA 6,9). In the EC condition, the localization is left dominant (delta and theta) or bilateral (beta) (Fig. 4). In 


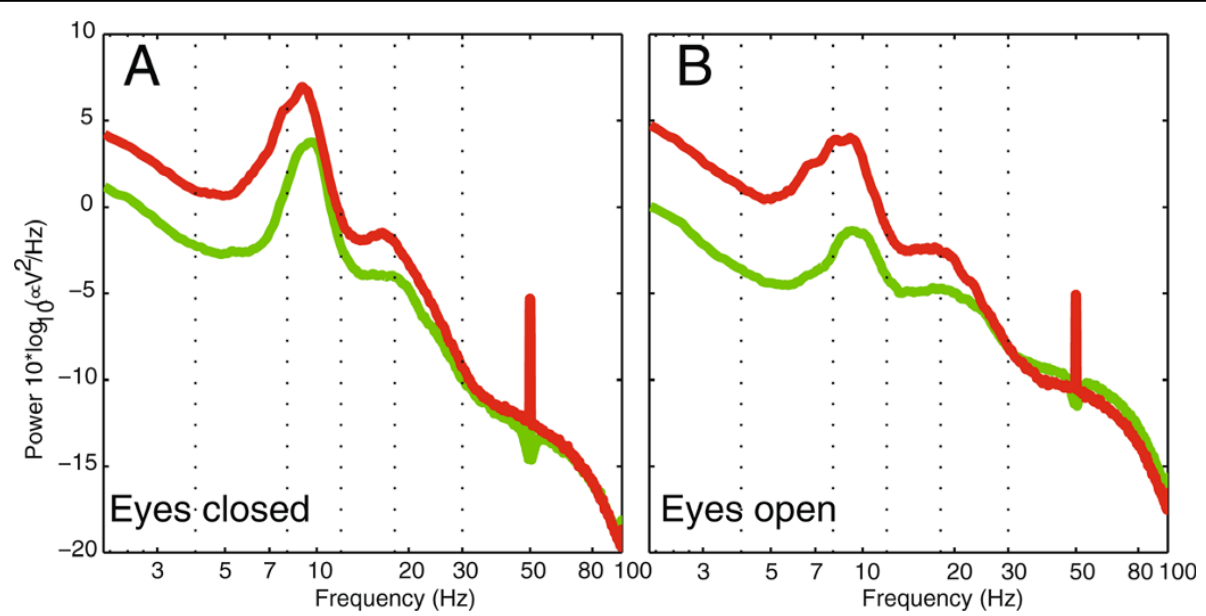

Figure 1 Enhanced EEG power in tinnitus patients. In global EEG power spectrum for the group of patients (red) was enhanced with respect to the group of healthy controls (green) in A and B for EC and EO conditions respectively.
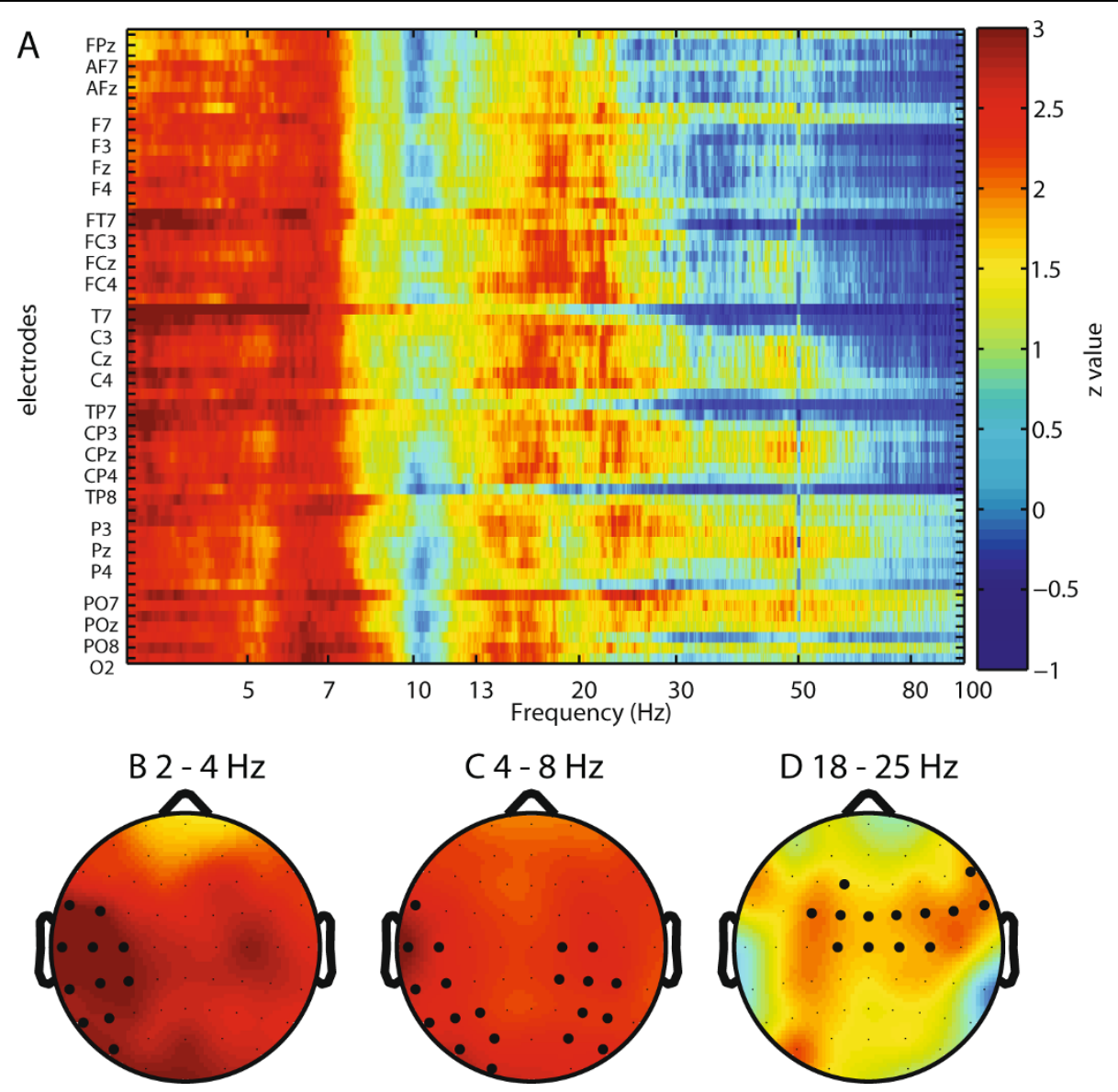

Figure 2 Electrodewise comparison of power spectra for EC condition. (A) Shown are Z-values for each electrode and frequency point (Wilcoxon rank sum tests). Non-corrected $Z$ values above 1.96 correspond to $p<0.05$. Highly significant electrodes were marked with black circles at different $p$ values. (B) Delta (2-4 Hz) band (corrected, $p<0.004)$. (C) Theta (4-8 Hz) band (corrected, $p<0.016)$. (D) Beta (18-25 Hz) band (corrected, $p<0.05$ ). 

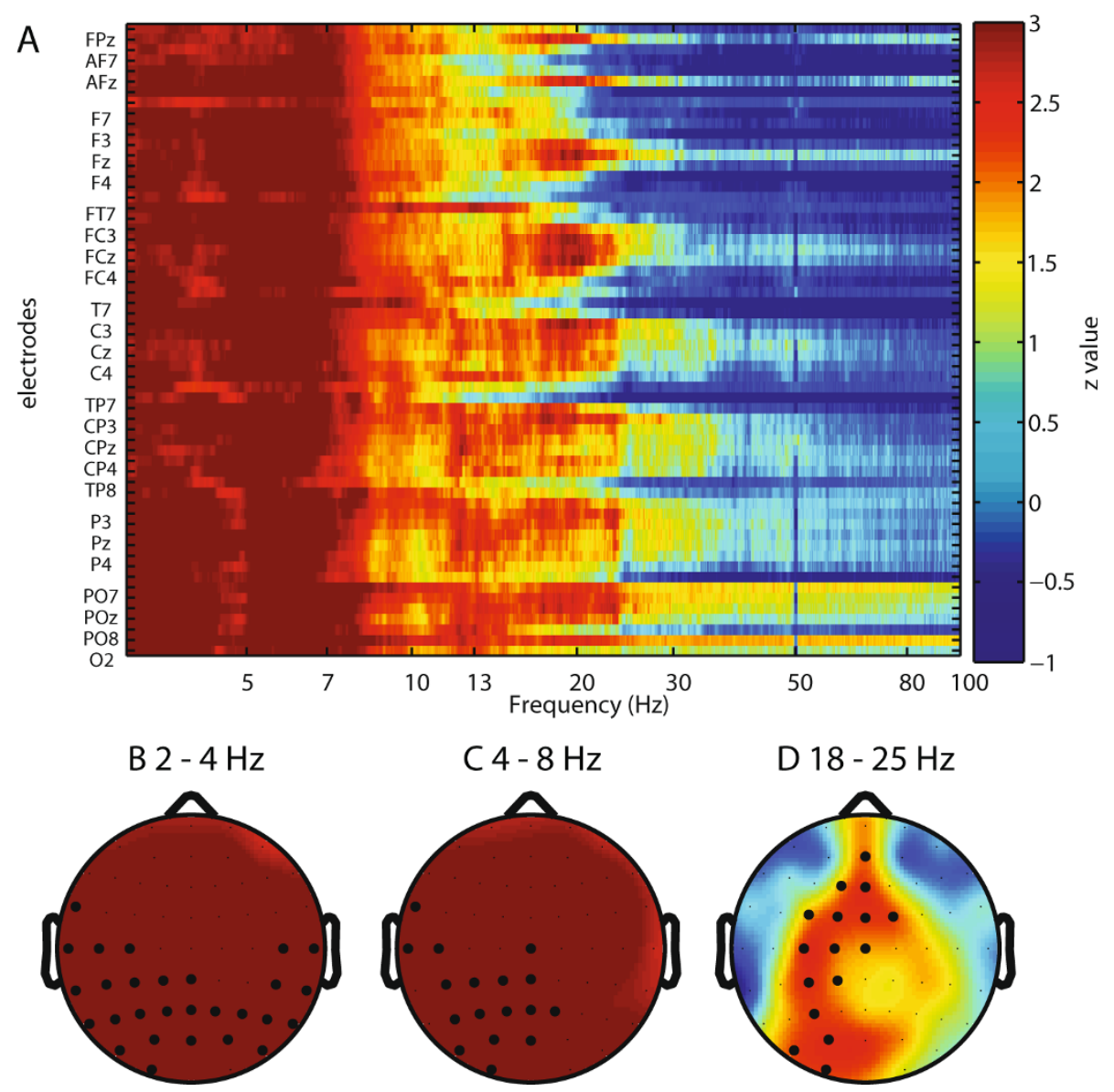

Figure 3 Electrodewise comparison of power spectra for EO condition. (A) Shown are Z-values for each electrode and frequency point (Wilcoxon rank sum tests). Non-corrected $Z$ values above 1.96 correspond to $p<0.05$. Highly significant electrodes were marked with black circles at different $p$ values. (B) Delta $(2-4 \mathrm{~Hz}$ ) band (corrected, $\mathrm{p}<0.0019)$. (C) Theta (4-8 Hz) band (corrected, $\mathrm{p}<0.001)$. (D) Beta (18-25 Hz) band (corrected, $\mathrm{p}<0.05$ ).

the EO condition, it is bilateral (delta) or left dominant (theta, alpha, and beta) (Fig. 5).

\section{Discussion and Conclusions \\ Power}

Our study on chronic tinnitus shows power enhancement of the spontaneous EEG activity, and localizes corresponding cortical generators of this dysrhythmic activity.

Our most striking finding in the EEG spectra of the patient group is the delta and theta EEG power enhancement (Fig. 1). An increase of cortical activity is in line with a previous MEG report of excess power in the whole frequency range for positive symptoms [7] and an increase of delta in tinnitus patients compared to healthy controls $[25,36]$. It is at variance with an earlier report [38]. This variance may be due to 1 ) the size of the patient groups and/or the selection of patients, and 2) the specific choice of frequency bands entering the statistical analysis. Our analysis is less likely to be biased in this respect, since Z-values are calculated for each frequency point. Concerning the statistics for band powers, we have corrected for multiple comparisons using FDR, yielding more potent statistical inference to our study.

The general increase in power in delta, theta and beta frequency ranges is confirmed if individual electrodes are analysed (Fig. 2A and Fig. 3A). This increase is generalized for theta and delta, and limited to fronto-centro-parietal sites in the beta domain. An increase of alpha is also found in the EO condition, localized on similar areas as delta and theta and indicating thus a participation of this frequency band in the thalamocortical dysrhythmia (TCD) tinnitus process. An increase in low and high frequencies is in line with the concept of TCD and may reflect the outspoken and chronic TCD involvement of our patient group [7,10,24,56]. This may explain the differences with the study of Weisz et al. 


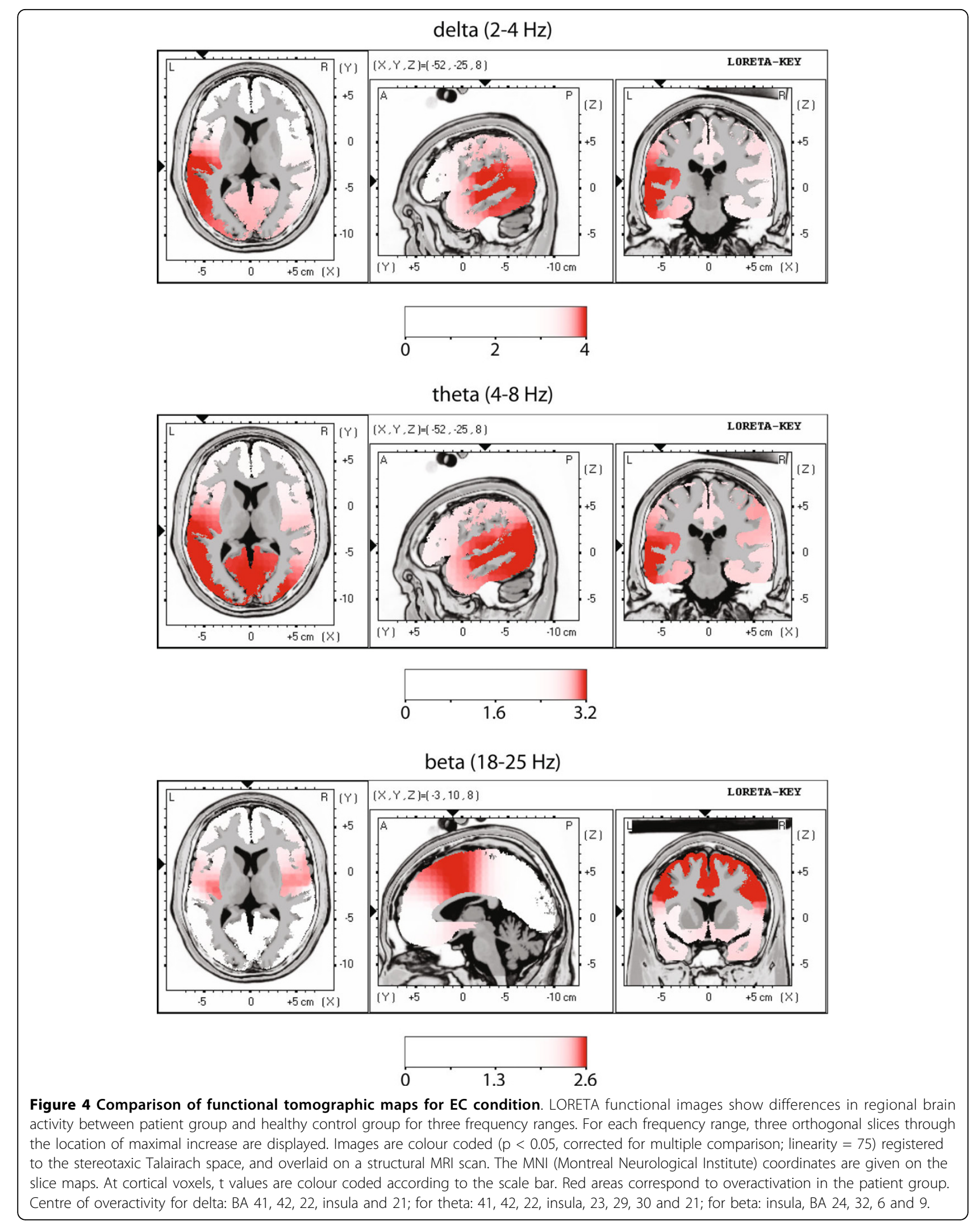



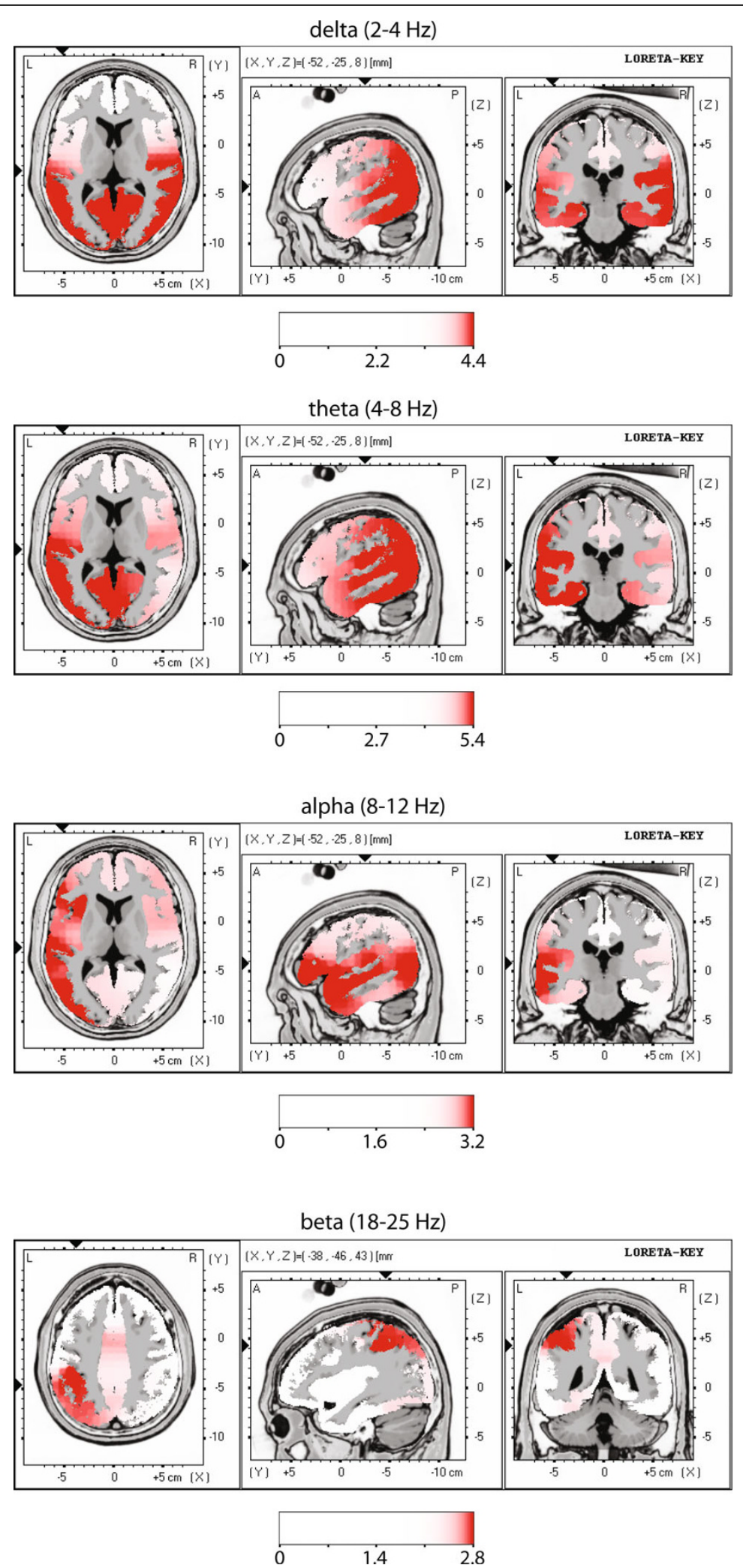

Figure 5 Comparison of functional tomographic maps for EO condition for four frequency bands. Center of overactivity for delta: BA 41, $42,22,23,29,30$, insula, 21, parahippocampal gyrus; for theta: BA 41, 42, 22, 23, 29, 30, insula, 21, parahippocampal gyrus; for alpha: BA 41, 42, 22, insula, 21, prefrontal; for beta: BA 40. 
[25], where only delta increase and a reduction of alpha were observed. Reduction of delta band power [16] and theta band power [57] after neurofeedback speaks for a causal relevance of slow oscillation increase in tinnitus. In an MEG case study, Llinas et al. [24] obtained a general (delta to beta) spectral power reduction after tinnitus masking.

\section{Cortical generators of excess EEG power}

In the delta, theta, alpha and beta bands, the cortical generators of excess EEG power were located in dominantly left auditory (BA 41, 42, 22) temporo-parietal, insular posterior, cingulate anterior and parahippocampal cortical areas. Such a localization is in accordance with data from metabolic studies [58-63] and speaks for a dysrhythmic co-involvement of associative and paralimbic areas in the pathogenesis of tinnitus, which is consistent with their topographic and functional vicinity with the auditory system. Many other studies pointed out the involvement of associative/paralimbic areas $[20,58,59,64]$ and the importance of reactive emotional factors in tinnitus has been repeatedly $[1,17,34,61]$ reported, as well as reciprocal involvement of auditory and associative/paralimbic areas [12,61]. We observe a dominance on the left side for both TCD and tinnitus. At the time, we can only speculate that this observation is related to cognitive/emotional factors, e.g. the relevance of the non-acceptance of, and related frustration about, the presence of tinnitus. This non-acceptance may be viewed as primarily conceptual, i.e. left-side dominant. (see below the cognitive/emotional factor).

\section{Thalamocortical Dysrhythmia}

Following the proposition of Llinas $[7,24,65]$ of a central relevance of $\mathrm{TC}$ interaction in the genesis of hemispheric function and encouraged by our earlier finding of strong TC coupling $[66,67]$, we propose here an interpretation of our results in the framework of TCD. This TC concept for neurogenic pain, abnormal movements, epilepsy, tinnitus and neuropsychiatric disorders was proposed $[7,56,68]$ on the basis of experimental $[26,28,29,69]$, and clinical evidence in the mentioned diseases [10]. It may be characterized by the following sequential set of events (schematized in Fig. 6):

(1) A lesion leads to deafferentation of excitatory inputs on thalamic relay cells and initiates the tinnitus syndrome (Fig. 6A). The deafferentation of excitatory inputs results in disfacilitation and cell membrane hyperpolarization.

(2) In the hyperpolarized state, deinactivation of calcium $\mathrm{T}$-channels causes thalamic relay neurons to fire LTS bursts at delta/theta frequency [26].

(3) Bursting thalamic relay neurons exert a rhythmic influence on $\mathrm{TC}$ loops in the delta/theta frequency band. Thalamic and cortical areas are densely and reciprocally interconnected $[29,30]$. The tight functional coupling between thalamus and cortex is confirmed by the high theta coherence between the two $[66,67]$. This coupling is sustained by thalamocorticothalamic and also by thalamoreticulothalamic and corticoreticulothalamic recurrent projections [28]. The tendency of the TC network to maintain a given functional modality reinforces the hyperpolarized state over time [70].

(4) Divergent TC, corticothalamic and reticulothalamic projections provide the anatomical substrate for diffusion of low frequency activity to an increasing number of neighbouring TC loops (Fig. 6B: theta cross-modular spread).

(5) After recruitment of a sufficiently large number of TC loops, excess delta/theta power becomes measurable. Increased low-frequency oscillations also occur during sleep [28] and cognitive tasks [71,72], where they are considered as normal. It is the continuous, widespread and state-independent overproduction of slow rhythms in the awake brain that characterizes TCD.

(6) The final step towards the production of tinnitus is related to the reciprocal cortico-cortical inhibition mediated by GABAergic interneurons, which is a general feature of cortical organization (Fig. 6C). TC modules in delta/theta mode exert less collateral inhibition on neighboring modules, which are thereby overactivated in high (beta/gamma) frequencies. This event has been termed "edge effect" [7]. The concept is inspired by the effect of lateral inhibition in the retina. It has also been considered in the periphery of the auditory system [1]. The asymmetrical inhibition between a low frequency cortical area and neighbouring high frequency domains provides a ring of reduced inhibition onto, and thus activation of, the cortex surrounding this low frequency area. Support for such an effect was first provided by the increased interfrequency covariation between theta and beta ranges in MEG [7]. Recently, the increase of high frequency activation around a core of theta modules could be demonstrated in a slice preparation [24].

Plasticity mechanisms have been proposed to be at the base of the appearance of tinnitus. The following observations speak however for the necessity of at least another mechanism. The presence of a deficit after a damage to the auditory system and the induction of plastic mechanisms are both seen to happen in all deafferentation situations, whereas tinnitus, quite like neurogenic pain, develops and maintains itself along years only in a small (less than 10\%) percentage of all deafferented subjects. Plastic map reorganizations, as argued by Weisz and collaborators [73], certainly contribute to the new post-lesional situation, but cannot satisfactorily explain the emergence and maintenance of tinnitus. 


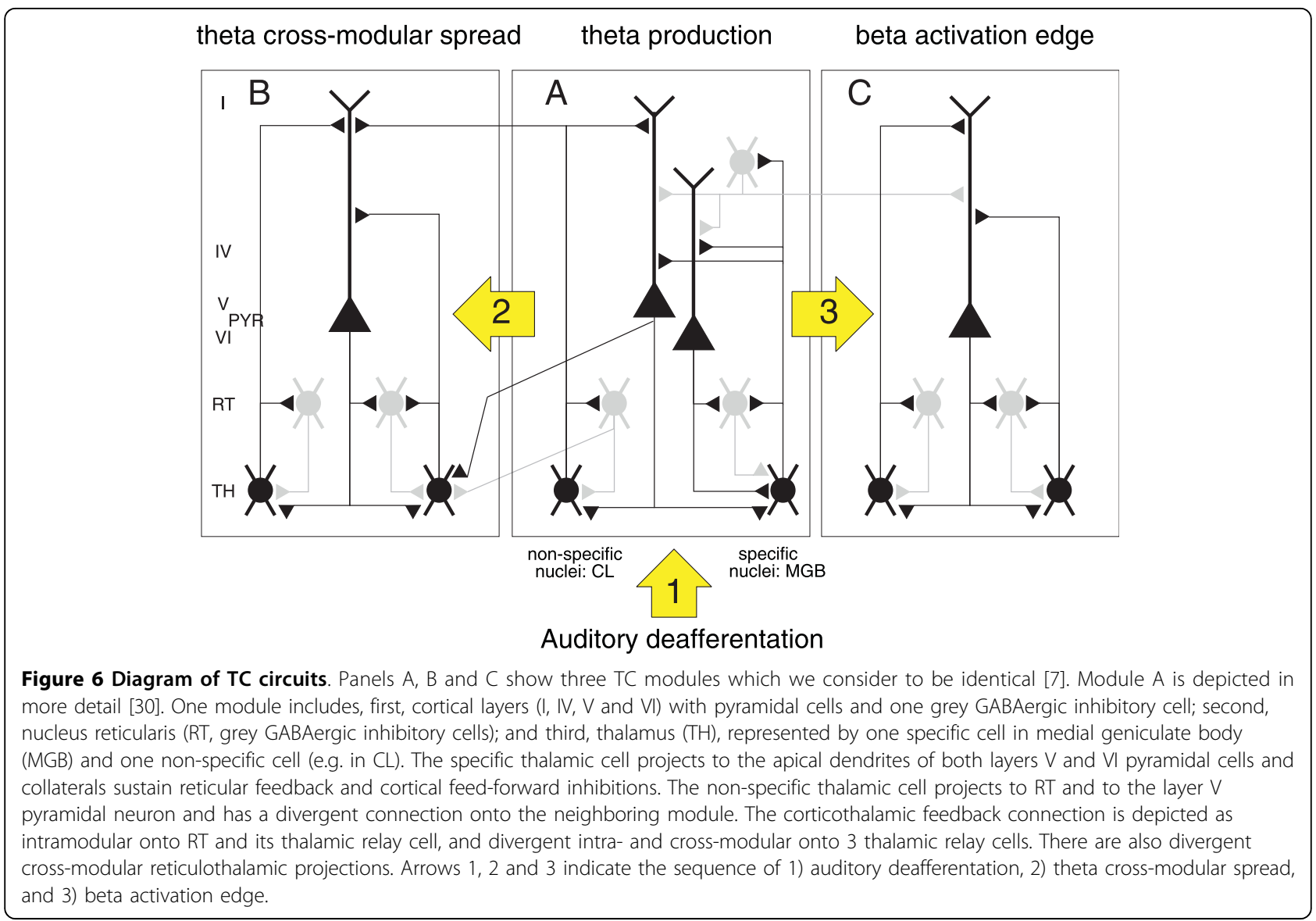

\section{The cognitive/emotional factor}

Accumulating evidence from EEG and MEG studies underscores the fact that conceptual, mnestic [71,74,75] and emotional [76] activations increase hemispheric theta activity. Our EEG source localization data confirm other studies demonstrating the coactivation of cortical auditory and associative/paralimbic areas. This provides a substrate for a role of mental functions in the reactive modulation of neurogenic (i.e. due to auditory deafferentation) tinnitus and the generation of psychogenic tinnitus. Indeed, a top-down activation of the divergent corticothalamic projection from associative/paralimbic onto auditory areas, when strong and long-lasting enough to cause sustained thalamic overinhibition, might be at the source of an increase of theta production and, thus, of an amplification of neurogenic tinnitus mechanisms. It may even be at the origin of psychogenic tinnitus. One of the most deleterious conceptual/emotional dynamics is, in our experience, frustration and non-acceptance of the disease-related health impairment. The conceptual and practical consequences of these considerations are obvious and have a wide range of implications for the therapeutic support of neurogenic and psychogenic tinnitus patients. These include the recognition of a dual origination of tinnitus (neurogenic and psychogenic), in body and mind, respectively, but the existence of a common TC mechanism for both.

Additional file 1: Table S1. Patient clinical details. Symptom laterality: BL (bilateral), R (right), L (left). Quality of Life: QL. Intensity scaling for anxiety, depression, frustration, hyperacusis and reduction of QL: 0 is for normal, 1 for slight, 2 for moderate, 3 for strong and 4 for severe. VAS: visual analogue scale (0-100). NA: not available.

Additional file 2: Grand average of the normalized power spectrum for EC condition in tinnitus and healthy control groups. Normalized EEG power spectrum for the group of patients (red) was enhanced with respect to the group of healthy controls (green).

\section{List of abbreviations}

BA: Brodmann area; CL: central lateral nucleus; EC: eyes closed; EO: eyes open; EEG: electroencephalogram; EMG: electromyogram; FDR: false discovery rate; FFT: fast Fourier transform; ICA: independent component analysis; LORETA: low resolution electromagnetic tomography analysis; LTS: low-threshold calcium spike; MEG: magnetoencephalogram; RI: residual inhibition; TC: thalamocortical; TCD: thalamocortical dysrhythmia.

\section{Acknowledgements}

We thank Jim Dodd for help with EEG recordings, Dr. Roberto PascualMarqui for help with LORETA. We gratefully acknowledge financial support by the Neuroscience Center Zurich, Focused Ultrasound Surgery Foundation and by the Roche Research Foundation. 


\section{Author details}

'Institute of Neuroinformatics, ETHZ/UNIZH, Winterthurerstrasse 190, 8057 Zurich, Switzerland. 'University Hospital Zurich, Laboratory for Functional Neurosurgery, CH-8091 Zurich, Switzerland. ${ }^{3}$ Department of Psychology, University of Konstanz, D-78464 Konstanz, Germany. ${ }^{4}$ Center for Integrative Human Physiology, University of Zurich, CH-0857 Zurich, Switzerland.

\section{Authors' contributions}

MMG conceived of the study, designed it, recorded the data, performed the data analysis, interpreted the data and drafted the manuscript. LM recorded the data and interpreted the data, NN recorded the data and interpreted of the data, DJ interpreted the data, drafted the manuscript and revised the manuscript critically. All authors read and approved the final manuscript.

Received: 7 September 2009 Accepted: 24 March 2010

Published: 24 March 2010

\section{References}

1. Jastreboff PJ: Phantom auditory perception (tinnitus): mechanisms of generation and perception. Neurosci Res 1990, 8(4):221-254.

2. Heller AJ: Classification and epidemiology of tinnitus. Otolaryngologic clinics of North America 2003, 36:239-248.

3. Hoffman H, Reed G: Tinnitus: Theory and Management. Epidemiology of tinnitus Lewiston, NY: B C DeckerSnow JB 2004, 16-41.

4. Eggermont JJ, Roberts LE: The neuroscience of tinnitus. Trends Neurosci 2004, 27(11):676-682

5. Norena A, Micheyl C, Chery-Croze S, Collet L: Psychoacoustic characterization of the tinnitus spectrum: implications for the underlying mechanisms of tinnitus. Audiology \& neuro-otology 2002, 7:358-369.

6. Weisz N, Hartmann T, Dohrmann K, Schlee W, Norena A: High-frequency tinnitus without hearing loss does not mean absence of deafferentation. Hearing Research 2006, 222(1-2):108-114.

7. Llinás R, Ribary U, Jeanmonod D, Kronberg E, Mitra PP: Thalamocortical dysrhythmia: A neurological and neuropsychiatric syndrome characterized by magnetoencephalography. Proc Natl Acad Sci USA 1999, 96(26):15222-15227.

8. Lockwood AH, Salvi RJ, Burkard RF: Tinnitus. N Engl J Med 2002, 347(12):904-910

9. Eggermont JJ: Central tinnitus. Auris Nasus Larynx 2003, 30(Suppl):S7-12

10. Jeanmonod D, Magnin M, Morel A: Low-threshold calcium spike bursts in the human thalamus. Common physiopathology for sensory, motor and limbic positive symptoms. Brain 1996, 119(Pt 2):363-375.

11. Reyes SA, Salvi RJ, Burkard RF, Coad ML, Wack DS, Galantowicz PJ, Lockwood AH: Brain imaging of the effects of lidocaine on tinnitus. Hear Res 2002, 171(1-2):43-50.

12. Eichhammer $P$, Hajak $G$, Kleinjung $T$, Landgrebe $M$, Langguth B, B Langguth GHTKACaARM: Functional imaging of chronic tinnitus: the use of positron emission tomography. Progress in Brain Research Elsevier 2007, 166:83-88

13. Shulman A, Goldstein B: A Translational Concept for Diagnosis and Treatment. Int Tinnitus J 2006, 2(12):101-114.

14. Shulman A, Seitz M: Central tinnitus - Diagnosis and treatment: Observations simultaneous auditory brainstem responses with monaural stimulation in the tinnitus patient. Laryngoscope 1981, 91:2025-2035.

15. Norena AJ, Eggermont JJ: Changes in spontaneous neural activity immediately after an acoustic trauma: implications for neural correlates of tinnitus. Hear Res 2003, 183(1-2):137-153.

16. Kahlbrock N, Weisz N: Transient reduction of tinnitus intensity is marked by concomitant reductions of delta band power. BMC Biol 2008, 6:4.

17. Weisz N, Voss $S$, Berg P, Elbert T: Abnormal auditory mismatch response in tinnitus sufferers with high-frequency hearing loss is associated with subjective distress level. BMC Neurosci 2004, 5:8,

18. Weisz N, Dohrmann K, Elbert T: The relevance of spontaneous activity for the coding of the tinnitus sensation. Prog Brain Res 2007, 166:61-70

19. Flor H, Elbert T, Knecht S, Wienbruch C, Pantev C, Birbaumer N, Larbig W, Taub E: Phantom-limb pain as a perceptual correlate of cortical reorganization following arm amputation. Nature 1995, 375(6531):482-484

20. Lockwood AH, Salvi RJ, Coad ML, Towsley ML, Wack DS, Murphy BW: The functional neuroanatomy of tinnitus: evidence for limbic system links and neural plasticity. Neurology 1998, 50(1):114-120.
21. Tonndorf J: The analogy between tinnitus and pain: a suggestion for a physiological basis of chronic tinnitus. Hear Res 1987, 28(2-3):271-275.

22. Moller AR: Similarities between chronic pain and tinnitus. Am J Otol 1997 18(5):577-585.

23. Moller AR: Similarities between severe tinnitus and chronic pain. J Am Acad Audiol 2000, 11(3):115-124.

24. Llinas R, Urbano FJ, Leznik E, Ramirez RR, van Marle HJ: Rhythmic and dysrhythmic thalamocortical dynamics: GABA systems and the edge effect. Trends Neurosci 2005, 28(6):325-333.

25. Weisz N, Moratti S, Meinzer M, Dohrmann K, Elbert T: Tinnitus perception and distress is related to abnormal spontaneous brain activity as measured by magnetoencephalography. PLoS Med 2005, 2(6):e153.

26. Llinás $\mathrm{R}$, Jahnsen $\mathrm{H}$ : Electrophysiology of mammalian thalamic neurones in vitro. Nature 1982, 297(5865):406-408.

27. Jahnsen $H$, Llinas $R$ : Voltage-dependent burst-to-tonic switching of thalamic cell activity: an in vitro study. Arch Ital Biol 1984, 122(1):73-82.

28. Steriade M: Impact of network activities on neuronal properties in corticothalamic systems. J Neurophysiol 2001, 86(1):1-39.

29. Steriade M, Jones EG, McCormick DA: Thalamus: Organisation and Function Oxford: Elsevier 1997, 1.

30. Jones EG: The thalamic matrix and thalamocortical synchrony. Trends Neurosci 2001, 24(10):595-601.

31. Llinas RR, Leznik E, Urbano FJ: Temporal binding via cortical coincidence detection of specific and nonspecific thalamocortical inputs: a voltagedependent dye-imaging study in mouse brain slices. Proc Natl Acad Sci USA 2002, 99(1):449-454.

32. Nunez PL, Wingeier BM, Silberstein RB: Spatial-temporal structures of human alpha rhythms: theory, microcurrent sources, multiscale measurements, and global binding of local networks. Hum Brain Mapp 2001, 13(3):125-164

33. Weiler EWJ, Brill K, Tachiki KH: Quantitative electroencephalography and tinnitus: a case study. Int Tinnitus J 2000, 6(2):124-126.

34. Weisz N, Muller S, Schlee W, Dohrmann K, Hartmann T, Elbert T: The neural code of auditory phantom perception. J Neurosci 2007, 27(6):1479-1484.

35. Ashton H, Reid K, Marsh R, Johnson I, Alter K, Griffiths T: High frequency localised "hot spots" in temporal lobes of patients with intractable tinnitus: a quantitative electroencephalographic (QEEG) study. Neurosci Lett 2007, 426(1):23-28.

36. Shulman A, Goldstein B: Quantitative electroencephalography: preliminary report-tinnitus. Int Tinnitus J 2002, 8(2):77-86.

37. Shulman A, Avitable MJ, Goldstein B: Quantitative electroencephalography power analysis in subjective idiopathic tinnitus patients: a clinical paradigm shift in the understanding of tinnitus, an electrophysiological correlate. Int Tinnitus J 2006, 12(2):121-131.

38. Weiler EWJ, Brill K, Tachiki KH, Wiegand R: Electroencephalography Correlates in Tinnitus. Int Tinnitus J 2000, 6(1):21-24.

39. Dietrich V, Nieschalk M, Stoll W, Rajan R, Pantev C: Cortical reorganization in patients with high frequency cochlear hearing loss. Hear Res 2001, 158(1-2):95-101

40. Muhlnickel W, Elbert T, Taub E, Flor H: Reorganization of auditory cortex in tinnitus. Proc Natl Acad Sci USA 1998, 95(17):10340-10343.

41. Lockwood AH, Salvi RJ, Coad ML, Arnold SA, Wack DS, Murphy BW, Burkard RF: The functional anatomy of the normal human auditory system: responses to 0.5 and $4.0 \mathrm{kHz}$ tones at varied intensities. Cereb Cortex 1999, 9(1):65-76.

42. Giraud AL, Chery-Croze S, Fischer G, Fischer C, Vighetto A, Gregoire MC, Lavenne F, Collet L: A selective imaging of tinnitus. Neuroreport 1999, 10(1):1-5.

43. Lockwood AH, Wack DS, Burkard RF, Coad ML, Reyes SA, Arnold SA, Salvi RJ: The functional anatomy of gaze-evoked tinnitus and sustained lateral gaze. Neurology 2001, 56(4):472-480.

44. Landolt HP, Retey JV, Tonz K, Gottselig JM, Khatami R, Buckelmuller I, Achermann P: Caffeine attenuates waking and sleep electroencephalographic markers of sleep homeostasis in humans. Neuropsychopharmacology 2004, 29(10):1933-1939.

45. Delorme A, Makeig S: EEGLAB: an open source toolbox for analysis of single-trial EEG dynamics including independent component analysis. J Neurosci Methods 2004, 134(1):9-21.

46. Mitra PP, Pesaran B: Analysis of dynamic brain imaging data. Biophys $J$ 1999, 76(2):691-708. 
47. Benjamini Y, Hochberg Y: Controlling the False Discovery Rate: A Practical and Powerful Approach to Multiple Testing. Blackwell Publishing for the Royal Statistical Society 1995, 57:289-300.

48. Pascual-Marqui RD, Michel CM, Lehmann D: Low resolution electromagnetic tomography: a new method for localizing electrical activity in the brain. Int J Psychophysiol 1994, 18(1):49-65.

49. Pizzagalli D, Pascual-Marqui RD, Nitschke JB, Oakes TR, Larson CL, Abercrombie HC, Schaefer SM, Koger JV, Benca RM, Davidson RJ: Anterior cingulate activity as a predictor of degree of treatment response in major depression: evidence from brain electrical tomography analysis. Am J Psychiatry 2001, 158(3):405-415.

50. Zumsteg D, Friedman A, Wieser HG, Wennberg RA: Propagation of interictal discharges in temporal lobe epilepsy: Correlation of spatiotemporal mapping with intracranial foramen ovale electrode recordings. Clinical Neurophysiology 2006, 117(12):2615-2626.

51. Mazziotta J, Toga A, Evans A, Fox P, Lancaster J, Zilles K, Woods R, Paus T, Simpson G, Pike B, et al: A probabilistic atlas and reference system for the human brain: International Consortium for Brain Mapping (ICBM). Philosophical Transactions of the Royal Society of London Series B: Biological Sciences 2001, 356(1412):1293-1322

52. Lancaster JL, Woldorff MG, Parsons LM, Liotti M, Freitas CS, Rainey L, Kochunov PV, Nickerson D, Mikiten SA, Fox PT: Automated Talairach Atlas labels for functional brain mapping. Human Brain Mapping 2000, 10(3):120-131

53. Brett $\mathrm{M}$, Johnsrude IS, Owen AM: The problem of functional localization in the human brain. Nat Rev Neurosci 2002, 3(3):243-249.

54. Frei E, Gamma A, Pascual-Marqui R, Lehmann D, Hell D, Vollenweider FX: Localization of MDMA-induced brain activity in healthy volunteers using low resolution brain electromagnetic tomography (LORETA). Hum Brain Mapp 2001, 14(3):152-165.

55. Nichols TE, Holmes AP: Nonparametric permutation tests for functional neuroimaging: a primer with examples. Hum Brain Mapp 2002, 15(1):1-25.

56. Llinás R, Ribary U, Jeanmonod D, Cancro R, Kronberg E, Schulman J, Zonenshayn M, Magnin M, Morel A, Siegemund M: Thalamocortical dysrhythmia I. Functional and imaging aspects. Thalamus Related Syst 2001, 1:237-244.

57. Weiler EWJ, Brill K, Tachiki KH, Schneider D: Neurofeedback and quantitative electroencephalography. Int Tinnitus J 2002, 8(2):87-93.

58. Mirz F, Pedersen B, Ishizu K, Johannsen P, Ovesen T, Stodkilde-Jorgensen H, Gjedde A: Positron emission tomography of cortical centers of tinnitus. Hear Res 1999, 134(1-2):133-144.

59. Mirz F, Gjedde A, Sodkilde-Jrgensen H, Pedersen CB: Functional brain imaging of tinnitus-like perception induced by aversive auditory stimuli. Neuroreport 2000, 11(3):633-637.

60. Mirz F, Gjedde A, Ishizu K, Pedersen CB: Cortical networks subserving the perception of tinnitus-a PET study. Acta Otolaryngol Suppl 2000, 543:241-243.

61. Muhlau M, Rauschecker JP, Oestreicher E, Gaser C, Rottinger M, Wohlschlager AM, Simon F, Etgen T, Conrad B, Sander D: Structural brain changes in tinnitus. Cereb Cortex 2006, 16(9):1283-1288.

62. Shulman A: The Final Common Pathway for tinnitus- the medial temporal lobe system. Int Tinnitus J 1995, 1(2):115-126.

63. Shulman A, Strashun A, Afriyie M, Aronson F, Abel W, Goldstein B: Spect imaging of brain and Tinnitus - Neurotologic.Neurologic Implications. INt Tinnitus J 1995, 1(1):13-29.

64. Mahlke C, Wallhausser-Franke E: Evidence for tinnitus-related plasticity in the auditory and limbic system, demonstrated by arg3.1 and c-fos immunocytochemistry. Hear Res 2004, 195(1-2):17-34.

65. Llinas R, Ribary U, Contreras D, Pedroarena C: The neuronal basis for consciousness. Philos Trans R Soc Lond B Biol Sci 1998, 353(1377):1841-1849.

66. Sarnthein J, Jeanmonod D: High thalamocortical theta coherence in patients with neurogenic pain. Neuroimage 2008, 39(4):1910-1917.

67. Sarnthein J, Jeanmonod D: High Thalamocortical Theta Coherence in Patients with Parkinson's Disease. J Neurosci 2007, 27(1):124-131.

68. Jeanmonod D, Magnin M, Morel A, Siegemund M, Cancro R, Lanz M, Llinás R, Ribary U, Kronberg E, Schulman J, et al: Thalamocortical dysrhythmia II. Clinical and surgical aspects. Thalamus Related Syst 2001, 1:245-254
69. Ribary U, loannides AA, Singh KD, Hasson R, Bolton JP, Lado F, Mogilner A, Llinas R: Magnetic field tomography of coherent thalamocortical $40-\mathrm{Hz}$ oscillations in humans. Proc Natl Acad Sci USA 1991, 88(24):11037-11041.

70. Pedroarena C, Llinas R: Dendritic calcium conductances generate highfrequency oscillation in thalamocortical neurons. Proc Natl Acad Sci USA 1997, 94(2):724-728.

71. Klimesch W: EEG alpha and theta oscillations reflect cognitive and memory performance: a review and analysis. Brain Res Brain Res Rev 1999, 29(2-3):169-195.

72. Kahana MJ, Seelig D, Madsen JR: Theta returns. Curr Opin Neurobiol 2001, 11(6):739-744.

73. Weisz N, Wienbruch C, Dohrmann K, Elbert T: Neuromagnetic indicators of auditory cortical reorganization of tinnitus. Brain 2005, 128(Pt 11):2722-2731.

74. Sasaki K, Tsujimoto T, Nambu A, Matsuzaki R, Kyuhou S: Dynamic activities of the frontal association cortex in calculating and thinking. Neurosci Res 1994, 19(2):229-233.

75. von Stein A, Sarnthein J: Different frequencies for different scales of cortical integration: from local gamma to long range alpha/theta synchronization. Int J Psychophysiol 2000, 38(3):301-313.

76. Machleidt W, Gutjahr L, Mugge A: [Basic emotions. Phenomenology, psychodynamics, EEG spectral analysis]. Monogr Gesamtgeb Psychiatr Psychiatry Ser 1989, 57:1-251.

doi:10.1186/1471-2202-11-40

Cite this article as: Moazami-Goudarzi et al:: Temporo-insular enhancement of EEG low and high frequencies in patients with chronic tinnitus. QEEG study of chronic tinnitus patients. BMC Neuroscience 2010 11:40.

\section{Submit your next manuscript to BioMed Central and take full advantage of:}

- Convenient online submission

- Thorough peer review

- No space constraints or color figure charges

- Immediate publication on acceptance

- Inclusion in PubMed, CAS, Scopus and Google Scholar

- Research which is freely available for redistribution

Submit your manuscript at www.biomedcentral.com/submit
Ciomed Central 